\title{
Hadron cascades in CORSIKA 8
}

\section{Ralf Ulrich, ${ }^{a, *}$ Anatoli Fedynitch, ${ }^{b}$ Tanguy Pierog, ${ }^{a}$ Maximilian Reininghaus ${ }^{a, c}$ and Felix Riehn ${ }^{d, e}$ on behalf of the CORSIKA8 Collaboration}

(a complete list of authors can be found at the end of the proceedings)

${ }^{a}$ Institute for Astropartile Physics, Karlsruhe Institute of Technology, Germany

${ }^{b}$ Institute for Cosmic Ray Research, The University of Tokyo, Japan

${ }^{c}$ Instituto de Tecnologías en Detección y Astropartículas, Buenos Aires, Argentina

${ }^{d}$ Instituto Galego de Física de Altas Enerxías, Universidade de Santiago de Compostela, Spain

${ }^{e}$ Laboratory of Instrumentation and Experimental Particles, Lisbon, Portugal

E-mail: ralf.ulrich@kit.edu

\begin{abstract}
We present characteristics of hadronic cascades from interactions of cosmic rays in the atmosphere, simulated by the novel CORSIKA 8 framework. The simulated spectra of secondaries, such as pions, kaons, baryons and muons, are compared with the cascade equations solvers MCEq in air shower mode, and full 3D air shower Monte Carlo simulations using the legacy CORSIKA 7. A novel capability of CORSIKA 8 is the simulation of cascades in media other than air, widening the scope of potential applications. We demonstrate this by simulating cosmic ray showers in the Mars atmosphere, as well as simulating a shower traversing from air into water. The CORSIKA 8 framework demonstrates good accuracy and robustness in comparison with previous results, in particular in those relevant for the production of muons in air showers. Furthermore, the impact of forward $\rho^{0}$ production on air showers is studied and illustrated.
\end{abstract}

$37^{\text {th }}$ International Cosmic Ray Conference (ICRC 2021)

July 12th-23rd, 2021

Online - Berlin, Germany

\footnotetext{
*Presenter
} 


\section{Introduction}

The capabilities of the CORSIKA 8 framework [1] for particle cascade simulations are used to study the level of accuracy of hadron cascade simulations by comparing different approaches. Hadron cascades are in the core of cosmic ray air showers and are, thus, of high importance for the formation of the subsequent electromagnetic cascade as well as the muon and neutrino production in air showers [8]. For this work we are using the version icrc-2021-b of CORSIKA 8, which includes Sibyll2.3d [2] and QGSJetII.4 [3], as well as tracking in magnetic fields. Furthermore, we will exploit and illustrate the capabilities with non-air and exo showers, consistently developing in various materials other than the Earth atmosphere.

Some of the current capabilities of CORSIKA 8 are shown, and the resulting hadron cascades are compared in detail to other results and simulation programs. A general understanding of hadron cascades in the most systematic way possible will be one of the cornerstones to better understand uncertainties in air shower simulations and to further improve the quality of simulations. The work presented here makes use of the high degree of modularity allowed and encouraged inside CORSIKA 8 , which can be easily extended as needed to tackle a wide range of problems.

\section{High-energy models}

The high-energy interaction models Sibyll2.3d and QGSJetII.4 are simulated with CORSIKA 8 (release: icrc-2021-b), CORSIKA 7 [4] (v7.7410) and MCEq [5] (v1.2.1, including Sibyll2.3c). The Linsley approximation of the US-standard atmosphere is used to approximate the Earth atmosphere. Here, we focus on vertical, proton-induced showers at $E_{0}=10^{18} \mathrm{eV}$ without thinning. Only the
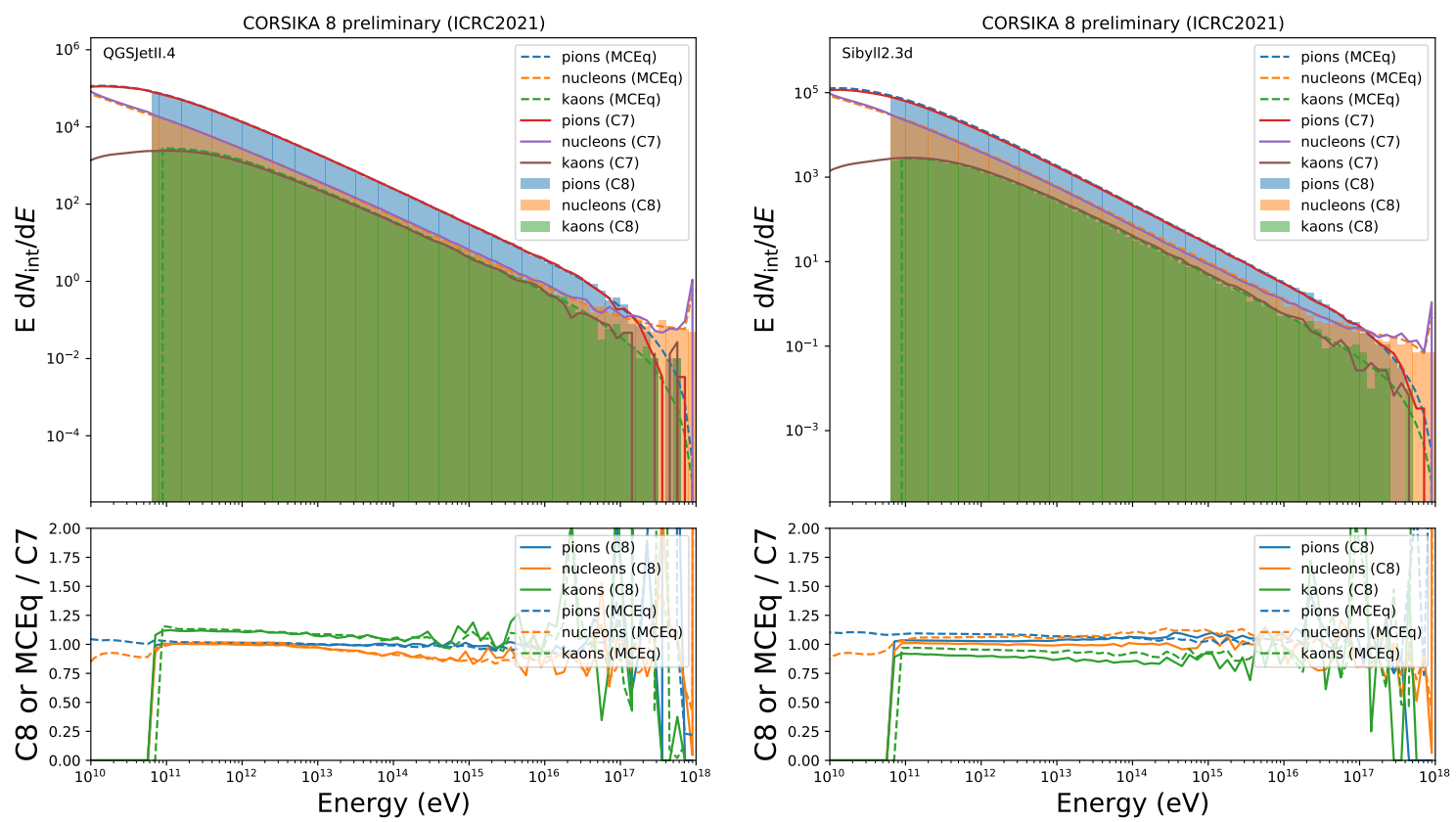

Figure 1: Total number of interaction as a function of projectile lab energy for various projectiles in the cascade for QGSJetII.4 (left) and Sibyll2.3d (right). Particles with kinetic energies above 63.1 GeV are shown. 

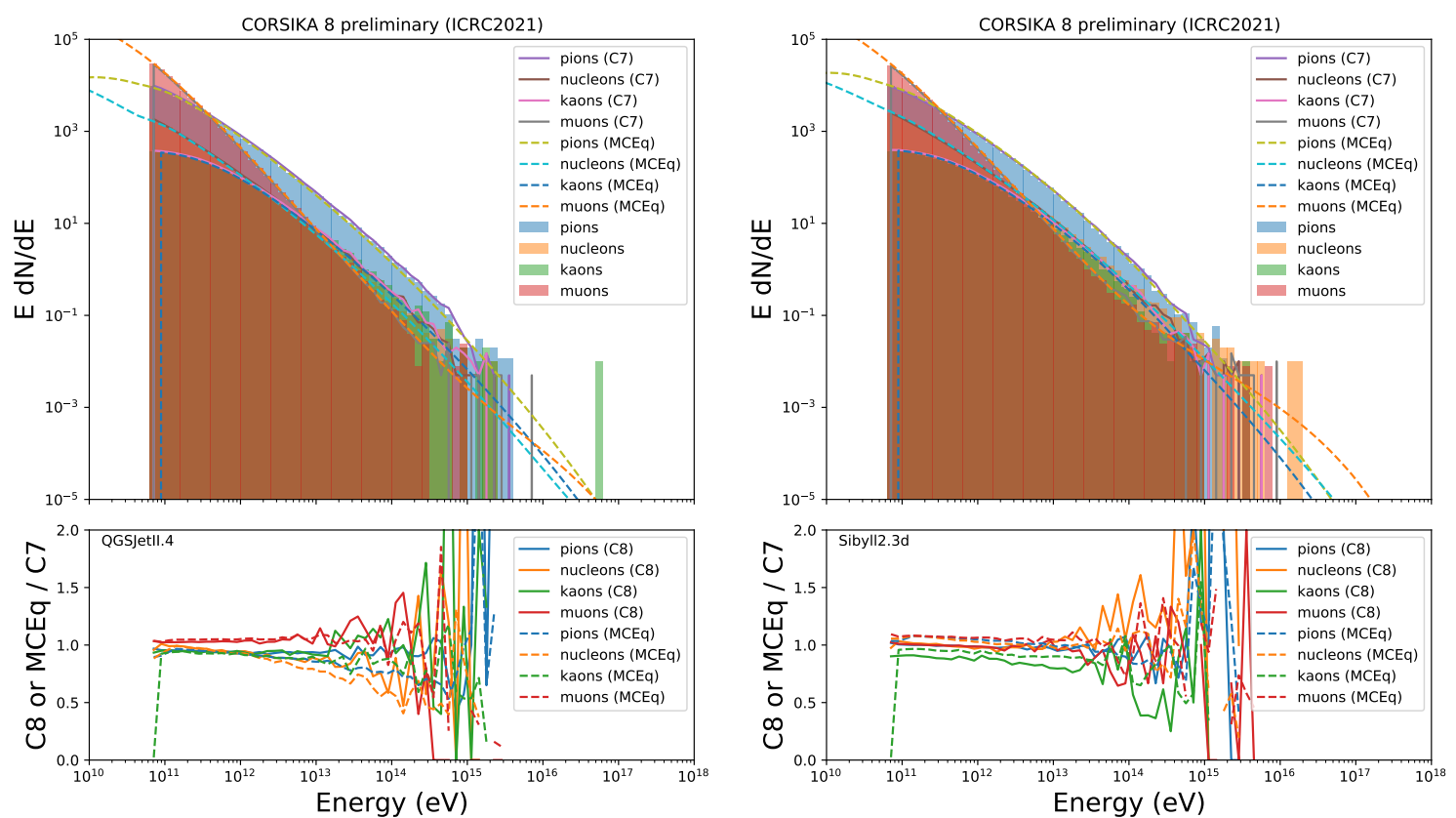

Figure 2: Secondary particle spectra at observation level (1400 m a.s.l) for QGSJetII.4 (left) and Sibyll2.3d (right).

high-energy models are used (for $E_{\text {lab }}>63.1 \mathrm{GeV}$ ), no low-energy model is used. Particles with energies below $63.1 \mathrm{GeV}$ are removed. For each result 200 showers are simulated and averaged.

One of the most critical distribution to study is the overall total energy distribution of collisions in the entire cascade. Each collision is responsible for multi-particle production, thus, deviations in the number of collisions have large impact on the final structure of the air shower. In Figure 1 these distributions are shown and compared to the ones of CORSIKA 7 as a reference. For QGSJetII.4 the agreement between CORSIKA 8 and MCEq is virtually perfect, while with CORSIKA 7 better than $\approx 10 \%$ with some energy-dependence. We note that for a model like QGSJetII.4 there is some freedom of interpretation in the interface to the model; we made an effort to synchronize our interface as much as possible with MCEq here.

For Sibyll2.3d the agreement of pions and nucleons with CORSIKA 7 is close to perfect, while there is a deficit of kaons on a level of $\approx 15 \%$. Note, that the MCEq version used here contains Sibyll2.3c with known deviations in particle production.

If we study the secondary particle spectra at Malargüe ground level $(1400 \mathrm{~m}$ above sea level corresponding to $875.5 \mathrm{~g} / \mathrm{cm}^{2}$ ) we find the results shown in Figure 2. The agreement is generally very good. Interestingly, the prediction for the muon energy spectrum comes out as the most robust prediction between all the investigated models. While for QGSJetII.4 the muon spectra ratios are completely energy independent, for Sibyll2.3d there is a very small energy dependence seen, which deserves further studies. Also there is a small but noticeable kaon deficit.

Moreover, the lateral arrival of secondary particles at the ground level was studied with CORSIKA 7 and CORSIKA 8. The results are shown in Figure 3 (left). There is an interesting systematic effect of a slightly wider shower footprint in CORSIKA 8 with respect to CORSIKA 7 visible. While this is a relatively small effect, it may well be important since detectors are often at far core distance and may notice this. However, the origin of this effect is not yet understood or 

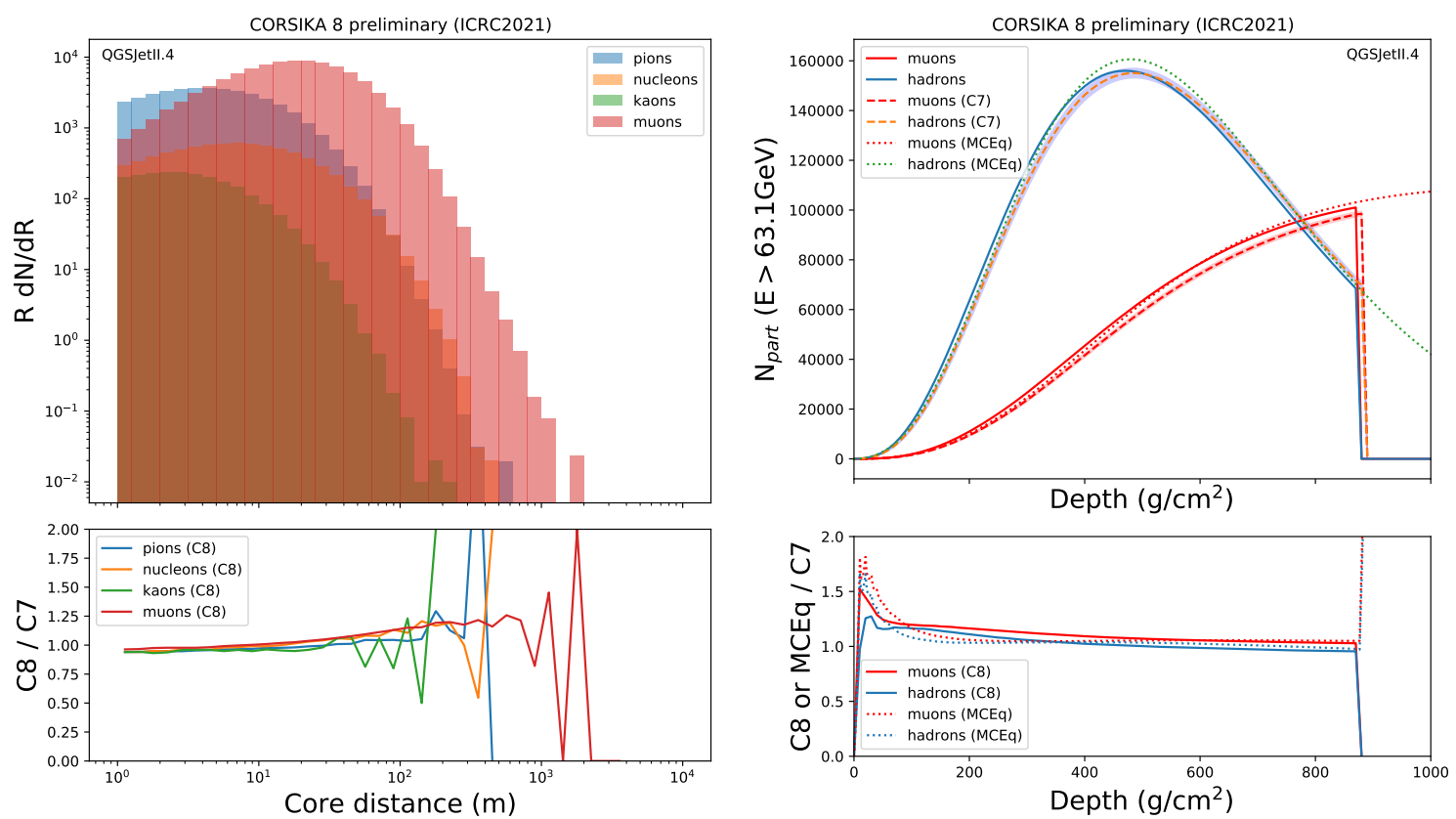

Figure 3: Left panel: Lateral particle number distribution for QGSJetII.4 comparing CORSIKA 8 and CORSIKA 7. Right panel: Longitudinal particle number profile for QGSJetII.4 comparing CORSIKA 8 to CORSIKA 7 and MCEq. Particles with kinetic energies above $63.1 \mathrm{GeV}$ are shown.

even fully studied - this will be further pursued by the CORSIKA 8 Collaboration.

Finally, in Figure 3 (right) we show the comparison of a longitudinal particle number profile for muons and hadrons, where the latter are the sum of $\pi^{ \pm}, \mathrm{K}_{\mathrm{L} / \mathrm{S}}^{0}, \mathrm{~K}^{ \pm}, \mathrm{n}, \mathrm{p}, \Lambda$ (and their anti-baryons). For CORSIKA 7 the uncertainty of the average profile is shown as a shaded band. The magnitude of this uncertainty depicts also an estimate of the uncertainty of the other profiles. The agreement is generally within statistical uncertainties, however, there are small systematic deviations visible consistent with the results shown above. CORSIKA 8 has a few more muons than CORSIKA 7. CORSIKA 8 indicates a general slightly slower shower development compared to MCEq and CORSIKA 8. MCEq has a slightly more narrow profile in general.

\section{Investigating of $\rho^{0} \leftrightarrow \pi^{0}$ conversion}

The particular importance of forward $\rho^{0}$ production in pion-air collision was found earlier [9, 10]. Following the method presented in Ref. [12] we developed a novel method for ad-hoc conversion of $\rho^{0} \leftrightarrow \pi^{0}$ in air showers. This was implemented inside the CORSIKA 8 framework and used to simulated the impact for various levels of conversion. The conversion factors are energy dependent and are limited to the interval $[-1,1]$ :

$$
p(E)=\operatorname{clamp}\left(p_{0}+\frac{f_{19}-p_{0}}{\log \frac{E_{\mathrm{th}}}{10^{19} \mathrm{eV}}} \max \left(0, \log \frac{E}{E_{\mathrm{th}}}\right)\right)
$$

where $\operatorname{clamp}(x)$ is defined as $\operatorname{clamp}(x)=\min (1, \max (-1, x))$. For $p(E)>0$ we replace $\pi^{0} \rightarrow \rho^{0}$ with probability $p(E)$. For negative values we replace $\rho^{0} \rightarrow \pi^{0}$ with probability $-p(E)$.

This method is applied to Sibyll2.3d proton showers. The effect is illustrated in Figure 4. While the electromagnetic $\mathrm{d} E /\left.\mathrm{d} X\right|_{\max }$ is anti-correlated with $f_{19}$ by a few percent, the muons at ground 

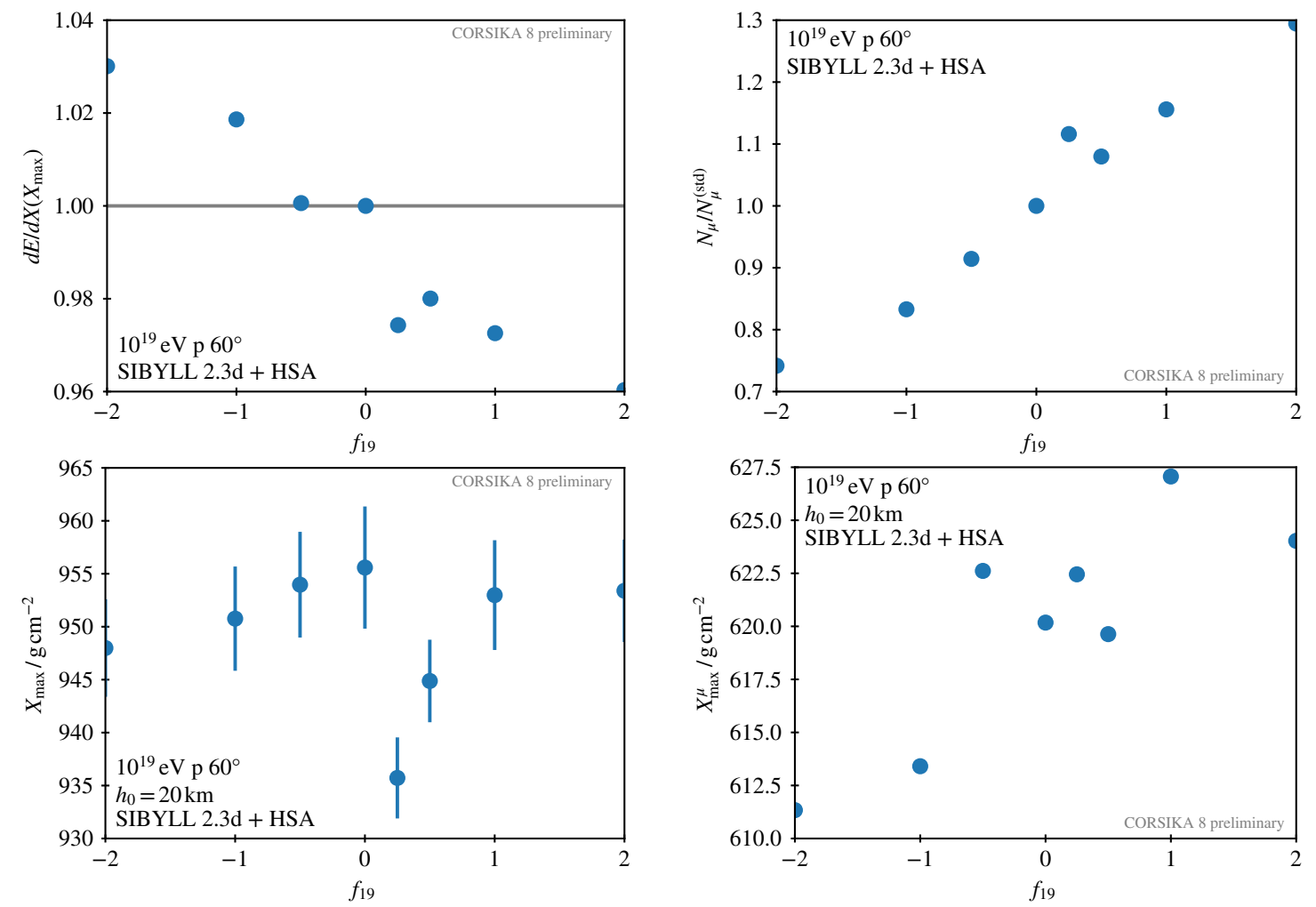

Figure 4: Overview of the results of converting $\rho^{0} \leftrightarrow \pi^{0}$ in air showers. Top left is $\mathrm{d} E /\left.\mathrm{d} X\right|_{\max }$ normalized to 1 at the reference point, top right is the muon number at ground, bottom left $X_{\max }$ and bottom right $X_{\max }^{\mu}$. Secondary particles with kinetic energies above $1 \mathrm{GeV}$ are shown.

are correlated and can change by tens of percent. At the same time, the longitudinal development of showers is hardly affected, both, $X_{\max }$ and also $X_{\max }^{\mu}$ are affected at most by a negligible degree.

For the work presented here the Hillas-splitting-algorithm (HSA) [14] as contained in AIRES [6] is used inside CORSIKA 8 for all kinetic lab energies below $63.1 \mathrm{GeV}$. Particles with kinetic energies below $1 \mathrm{GeV}$ are removed. Furthermore, all produced photons (and also few electrons) from hadron collisions are used as input for CONEX [7] 1D longitudinal profile calculations to derive a full energy deposit profile including $X_{\max }$. For this purpose, CONEX is run inside CORSIKA 8 as another physics module. This provides robust estimates of $\mathrm{d} E / \mathrm{d} X$ and, thus, $X_{\max }$.

\section{Non-standard showers}

The modularity and flexibility of CORSIKA 8 and its provided media-description facilities allow for the straightforward configuration of non-standard environments, thus, non-air or even exo-showers. Materials, geometries, magnetic fields, and in general: all properties of volumes, can be easily configured as needed by users.

\subsection{Exo-showers on Mars}

The integrated density column of the Mars atmosphere is just a few percent compared to Earth. Vertical showers see almost no material before they hit the ground. We implemented a two-layer 

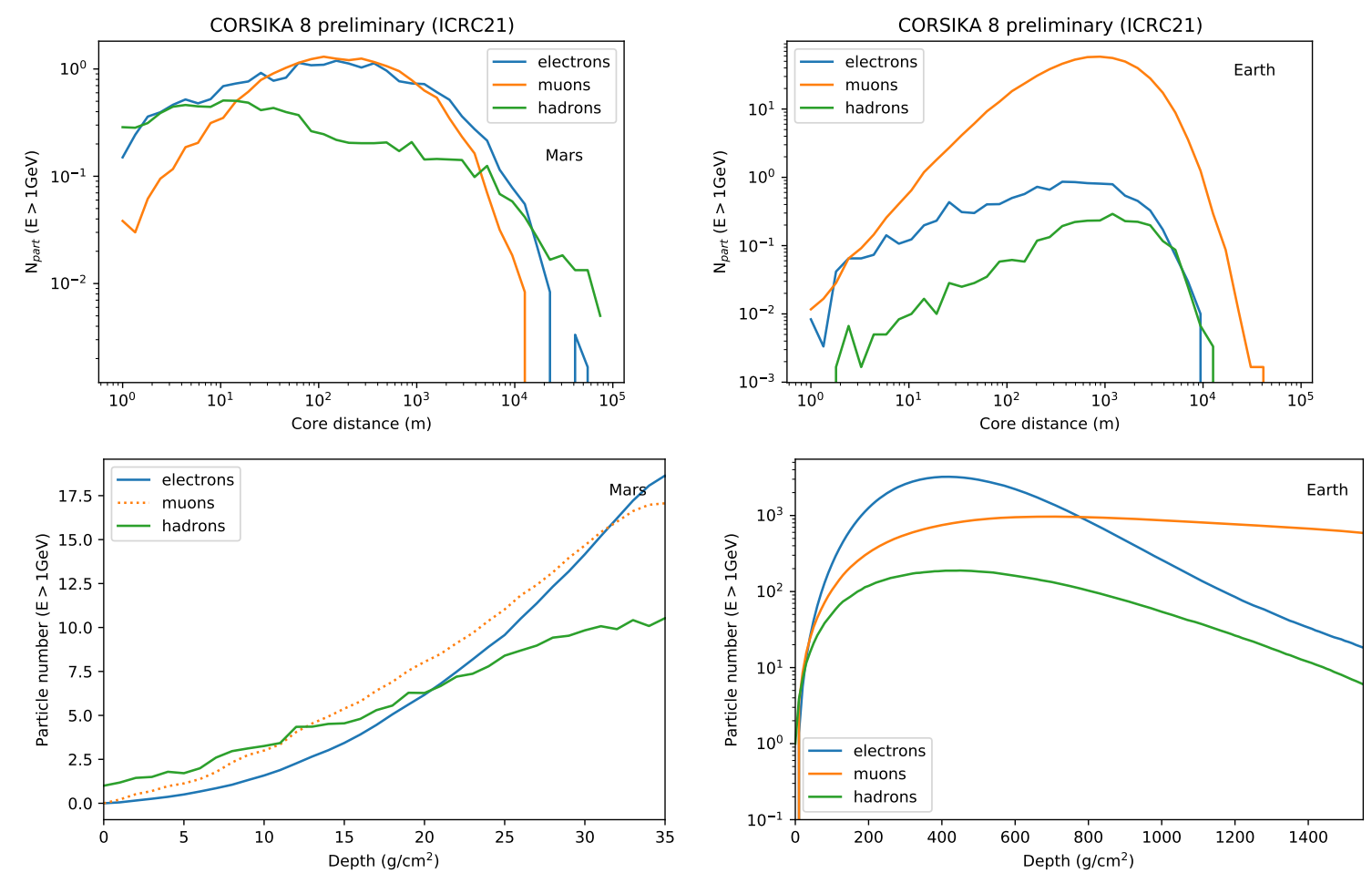

Figure 5: Summary of $100 \mathrm{TeV}$ proton induced showers on Mars (left) and Earth (right) at $60^{\circ}$ of zenith angle. On the top lateral particle number distributions on ground level are shown, while on the bottom this is shown for longitudinal profiles. Secondary particles with kinetic energy above $1 \mathrm{GeV}$ are shown.

Mars atmosphere and simulated inclined $\left(\theta=60^{\circ}\right)$ proton showers on Earth and on Mars with a $<1 \mathrm{GeV}$ particle cutoff. The low energy model is UrQMD with a transition at $E_{\text {kin,lab }}=63.1 \mathrm{GeV}$ to the high energy model Sibyll2.3d. Furthermore, E.m. interactions are fully simulated with PROPOSAL. Earth has a magnetic field strength of $50 \mu T$ and a standard Linsley atmosphere, while Mars has no magnetic field and a two layer density model provided by NASA [11]. Due to a current technical limitation of UrQMD in CORSIKA 8 the atmosphere composition of Mars is kept as $78 \%$ nitrogen and $22 \%$ oxygen instead of almost pure $\mathrm{CO}_{2}$. In the future this is not going to be necessary - but it will hardly affect the results shown here. The results in lateral and longitudinal direction are shown in Figure 5. Shown are the averages over 600 showers. Indeed, the showers on Mars are seen in very early stages of their development. It will be an interesting further study to include the Mars soil into the simulations to determine a full picture of the effect of cosmic radition on the surface of Mars.

\subsection{Showers with a transition from air to water}

When the observation level of CORSIKA 8 is moved to (negative) $3 \mathrm{~km}$ below sea level, the material between sea level and the observation level can be configured to be constant-density water with the correct, known, ionization energy loss parameters. Showers will first normally develop in air until the transition surface between air and water, and then consistently enter into water and continue their development towards the observation level. Of course, any backscatter from the dense medium into air is absolutely possible, too. The density model is shown in Figure 6 (left). 

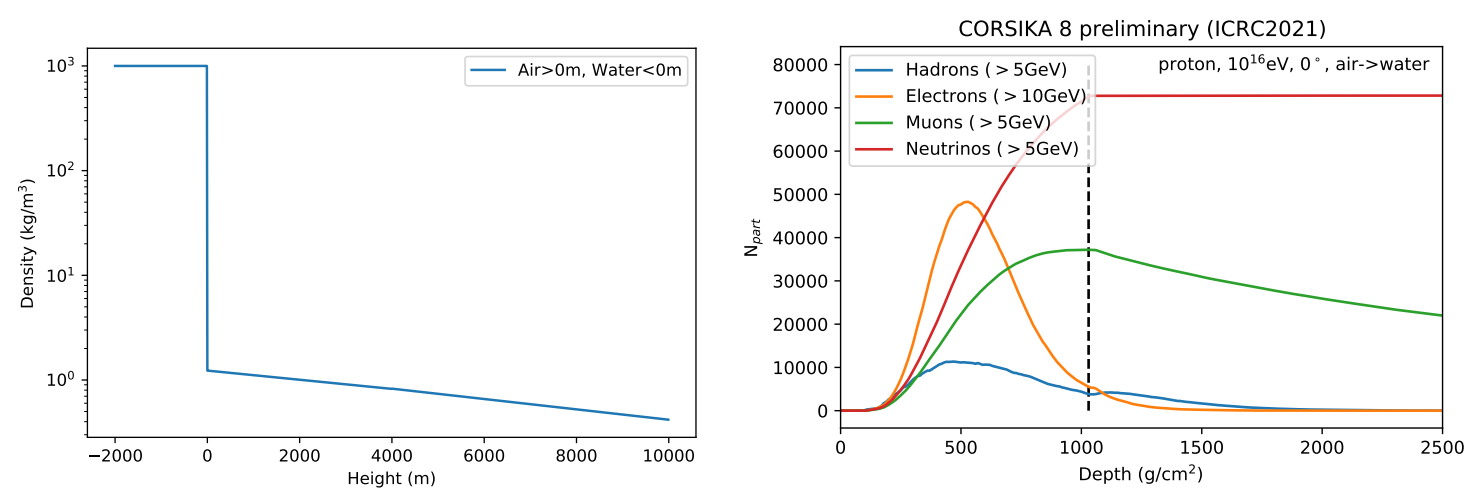

Figure 6: Left panel: Density model with a Linsley US std. atmosphere above sea level and water below sea level. Right panel: Longitudinal profile with a transition from air to water. The transition depth is shown as a dashed line. Note that the shown $1500 \mathrm{~g} / \mathrm{cm}^{2}$ of water depth just correspond to about $15 \mathrm{~m}$ of water column, while the simulation continues down to $-3 \mathrm{~km}$.

Here we use UrQMD for energies below $63.1 \mathrm{GeV}$ and Sibyll2.3 above this. E.m. interactions are handled with PROPOSAL. Particles with energies below $5 \mathrm{GeV}$ are removed; electrons and photons already at $10 \mathrm{GeV}$. Note, that currently PROPOSAL does not yet include density-dependent effects like LPM, but this will change in the future and will further improve such simulations.

In the resulting longitudinal particle number profile also neutrinos are included here, see Figure 6 (right), to illustrate the propagation through dense media. While the e.m. cascade is almost instantly absorbed in water, and muons start to be attenuated, the neutrinos are just continuing their trajectories to the observation level. It is interesting to note, that when the hadron core hits the dense media a very brief period of enhanced shower activity occurs - in distance this is just a few meters. While the properties in terms of ionization of water and air are just slightly different (e.g. critical energy in air is $88 \mathrm{MeV}$ and in water $78 \mathrm{MeV}$ ) the main difference is the density and thus the energy loss per meter being a factor of $\approx 1000$ higher in water. In combination, this leads to very efficient energy transport in water towards low energy particles (and below the cutoff energy of the simulation). In the longitudinal profiles this is evident since the regeneration of muons and also neutrinos in water above the energy cutoff is quite small. One can also imagine the conversion from depth to geometric distance to be very important. The decay of particles happens on geometric distance scale which is a factor of 1000 compressed in water compared to air, strongly suppressing the generation of new particles via decays per unit of depth.

\section{Summary}

CORSIKA 8 is becoming a highly versatile, fundamentally modular and powerful tool to perform particle cascade simulations. Sufficient completeness in term of provided models and robustness already allow for specific limited studies, many of which could not be done (easily) with other existing tools and programs. It was just started to perform real validation studies with CORSIKA 8, while in parallel more-and-more features and physics models are included.

In this work we have focused on a detailed comparison of air showers at high energies between different models and simulation programs. Preliminary results indicate agreement (mostly much) better than $10 \%$, with a few points worth further investigations. For example kaon spectra are 
a bit less consistently simulated, also there seem to be some small energy-dependent deviations. An indeed interesting result is that the LDF at sea level is slightly but systematically wider in CORSIKA 8 than in CORSIKA 7. Finally, longitudinal profiles simulated with CORSIKA 7 appear to be slightly slower in their development than, both, CORSIKA 8 and MCEq. This is even a bit more pronounced in MCEq than in CORSIKA 8.

In conclusion, CORSIKA 8 is shown to be already a partially powerful and robust tool for various novel and specific air shower physics studies. However, the framework is not yet ready for mass applications, further fundamental work is ongoing towards this direction. The project is open for collaborators and early users for testing, improvements, feedback and contributions [15].

\section{References}

[1] R. Engel et al.. Comput. Softw. Big Sci. 3 (2019) 2. doi:10.1007/s41781-018-0013-0. arXiv:1808.08226 [astro-ph.IM].

[2] F. Riehn, R. Engel, A. Fedynitch, T. K. Gaisser and T. Stanev. Phys. Rev. D 102 (2020) 063002. doi:10.1103/PhysRevD.102.063002. arXiv:1912.03300 [hep-ph].

[3] S. Ostapchenko. Phys. Rev. D 83 (2011) 014018. doi:10.1103/PhysRevD.83.014018. arXiv:1010.1869 [hep-ph].

[4] D. Heck, J. Knapp, J. N. Capdevielle, G. Schatz and T. Thouw. FZKA-6019.

[5] A. Fedynitch, H. Dembinski, R. Engel, T. K. Gaisser, F. Riehn and T. Stanev. PoS ICRC2017 (2018) 1019. doi:10.22323/1.301.1019.

[6] S. J. Sciutto. doi:10.13140/RG.2.2.12566.40002. arXiv:astro-ph/9911331 [astro-ph].

[7] T. Bergmann, et al. Astropart. Phys. 26 (2007) 420. doi:10.1016/j.astropartphys.2006.08.005. arXiv:astro-ph/0606564 [astro-ph].

[8] A. Fedynitch, M. Huber. Proceedings of 37th International Cosmic Ray Conference, PoS ICRC2021.

[9] H. J. Drescher. Phys. Rev. D 77 (2008) 056003. doi:10.1103/PhysRevD.77.056003. arXiv:0712.1517 [hep-ph].

[10] S. Ostapchenko. EPJ Web Conf. 52 (2013) 02001. doi:10.1051/epjconf/20125202001.

[11] https://www.grc.nasa.gov/www/k-12/airplane/atmosmrm.html.

[12] R. Ulrich, R. Engel and M. Unger. Phys. Rev. D 83 (2011) 054026. doi:10.1103/PhysRevD.83.054026. arXiv:1010.4310 [hep-ph].

[13] R. Ulrich, C. Baus and R. Engel. EPJ Web Conf. 99 (2015) 11001. doi:10.1051/epjconf/20159911001.

[14] A. M. Hillas. Nucl. Phys. B Proc. Suppl. 52 (1997) 29. doi:10.1016/S0920-5632(96)00847-X.

[15] gitlab.ikp.kit.edu/AirShowerPhysics/corsika. 


\section{Full Authors List: CORSIKA8 Collaboration}

Jean-Marco Alameddine ${ }^{b}$, Johannes Albrecht ${ }^{b}$, Jaime Alvarez-Muniz ${ }^{r}$, Antonio Augusto Alves $\mathrm{Jr}^{d}{ }^{\text {, Luisa Arrabito }}{ }^{a}$, Dominik Baack ${ }^{b}$, Konrad Bernlöhr $^{c}$, Marcus Bleicher ${ }^{o}$, Johan Bregeon ${ }^{s}$, Mathieu Carrere ${ }^{a}$, Hans Dembinski ${ }^{b}$, Hannah Elfner ${ }^{i}$, Dominik Elsässer ${ }^{b}$, Ralph Engel $^{d}$, Hu Fan ${ }^{p}$, Anatoli Fedynitch ${ }^{j}$, Dieter Heck ${ }^{d}$, Tim Huege ${ }^{d, e}$, Karl-Heinz Kampert ${ }^{k}$, Nikolaos Karastathis ${ }^{d}$, Lukas Nellen ${ }^{f}$, Maximilian Nöthe ${ }^{b}$, David Parello ${ }^{t}$, Tanguy Pierog $d$, Maria Pokrandt ${ }^{d}$, Anton Poctarev ${ }^{d}$, Remy Prechelt $^{l}$, Maximilian Reininghaus $^{d, m}$, Wolfgang Rhode ${ }^{b}$, Felix Riehn ${ }^{h, r}$, Maximilian Sackel ${ }^{b}$, Alexander Sandrock ${ }^{u}$, Pranav Sampathkumar ${ }^{d}$, Michael Schmelling $^{c}$, André Schmidt ${ }^{d}$, Günter Sigl ${ }^{n}$, Jan Soedingrekso ${ }^{b}$, Bernhard $\operatorname{Spaan}^{b}$, Donglian $\mathrm{Xu}^{q}$, Juan Ammerman-Yebra ${ }^{r}$ and Enrique $\mathrm{Zas}^{r}$

${ }^{a}$ Laboratoire Univers et Particules, Université de Montpellier 2, Montpellier, France, ${ }^{b}$ Experimentelle Physik 5, TU Dortmund, Dortmund, Germany, ${ }^{c}$ Max Planck Institute for Nuclear Physics, Heidelberg, Germany, ${ }^{d}$ Institute for Astroparticle Physics, Karlsruhe Institute of Technology, Karlsruhe, Germany, ${ }^{e}$ Astrophysical Institute, Vrije Universiteit Brussel, Brussels, Belgium ${ }^{f}$ National Autonomous University of Mexico, Mexico City, Mexico, ${ }^{g}$ Instituto de Tecnologías en Detección y Astropartículas, Buenos Aires, Argentina, ${ }^{h}$ Laboratory of Instrumentation and Experimental Particles, Lisbon, Portugal, ${ }^{i}$ Helmholtzzentrum für Schwerionenforschung, Darmstadt, Germany, ${ }^{j}$ Institute for Cosmic Ray Research, The University of Tokyo, Tokyo, Japan, ${ }^{k}$ Lehrstuhl für Astroteilchenphysik, Bergische Universität Wuppertal, Wuppertal, Germany, ${ }^{l}$ Department of Physics \& Astronomy, University of Hawai'i at Manoa, Honolulu, USA, ${ }^{m}$ Instituto de Tecnologías en Detección y Astropartículas, Buenos Aires, Argentina, ${ }^{n}$ II Institut für Theoretische Physik, Universität Hamburg, Hamburg, Germany, ${ }^{o}$ Johann-Wolfgang-Goethe-Universitaet, Frankfurt am Main, Germany, ${ }^{p}$ Peking University, Beijing, China, ${ }^{q}$ Tsung-Dao Lee Institute, Shanghai, China, ${ }^{r}$ Instituto Galego de Física de Altas Enerxías, Universidade de Santiago de Compostela, Santiago de Compostela, Spain, ${ }^{s}$ Laboratoire de Physique Subatomique et de Cosmologie, Grenoble, France, ${ }^{t}$ LIRMM, Univ Montpellier, CNRS, Montpellier, France, ${ }^{u}$ National Research Nuclear University, Moscow Engineering Physics Institute, Moscow, Russia. 